\title{
Les hussards des Alpes et le département de l'Ain
}

Jérôme Croyet

\section{(2) OpenEdition}

\section{Journals}

Édition électronique

URL : https://journals.openedition.org/ahrf/682

DOI : 10.4000/ahrf.682

ISSN : 1952-403X

Éditeur :

Armand Colin, Société des études robespierristes

Édition imprimée

Date de publication : 1 septembre 2002

Pagination : 67-88

ISSN : 0003-4436

\section{Référence électronique}

Jérôme Croyet, «Les hussards des Alpes et le département de l'Ain », Annales historiques de la

Révolution française [En ligne], 329 | juillet-septembre 2002, mis en ligne le 27 mars 2008, consulté le 23 avril 2022. URL : http://journals.openedition.org/ahrf/682 ; DOI : https://doi.org/10.4000/ahrf.682 


\title{
LES HUSSARDS DES ALPES ET LE DÉPARTEMENT DE L'AIN
}

\author{
JÉRÔME CROYET
}

\begin{abstract}
En l'an III, un corps franc créé par les représentants du peuple sur l'arrière de l'armée des Alpes, les hussards des Alpes, est employé dans l'ouest du département de l'Ain par les représentants du peuple à Lyon et les administrations locales afin de senvir comme troupes d'occupation pour accélérer le versement de grains dans les magasins militaires. Mais cette force devient rapidement une force de police afin d'empêcher le trafic des grains. Son utilité est alors valable le temps de sa présence, mais sur le long terme son passage n'aura d'autres conséquences que de marquer le souvenir des populations locales. Ce corps devenu régiment va, en effet, quitter l'Ain pour rejoindre l'armée et contribuer à la prestigieuse campagne d'Italie de 1796, à l'issue de laquelle, sous la pression de son colonel, il sera dissous.
\end{abstract}

Mots clés : Représentant du peuple ; hussard ; armée des Alpes ; subsistances ; Trévoux; Montluel ; Pont-de-Vaux.

Si l'an III de la République est marqué politiquement dans l'Ain par la réaction thermidorienne, il est aussi marqué par une grande vague de réquisitions militaires, puis par l'éloignement du front avec le commencement de la campagne d'Italie qui voit l'avènement d'un jeune général corse.

À l'automne 1794, les réquisitions de subsistances ont du mal à se faire dans le département de l'Ain, sur l'arrière de l'armée des Alpes. Pour les administrations, le trafic de grains avec la Suisse, par le Rhône, et les exportations illégales sabotent l'approvisionnement de Lyon et de l'armée. Pour les populations civiles, la rétention de grains est une mesure de survie, en ces temps où les réquisitions sont permanentes, quand il devient souvent bien difficile de nourrir sa famille. Pour certains cultivateurs et propriétaires, l'exportation et la vente de grains sur d'autres marchés sont une forme de revenu supplémentaire. Devant l'inertie des districts du sud du département de l'Ain, les hussards des Alpes, basés à Vienne sur les arrières 
de l'armée des Alpes, deviennent une force de police des représentants du peuple à Lyon qui utilisent ce corps franc comme un moyen de pression sur les populations afin qu'elles obéissent à leurs arrêtés. L'emploi d'un corps de hussards, plus que tout autre régiment, montre la volonté de marquer les esprits des populations civiles. En effet les hussards, troupe de cavalerie légère à l'allure chamarrée, n'en ont pas moins l'image de mauvais garçons caracolant en avant des armées, vivant de maraudes sur les terres conquises. Hommes de guerre par choix, les hussards sont de farouches combattants, centaures de l'armée, excellents dans l'indiscipline comme dans l'action. $\mathrm{Ne}$ disent-ils pas d'eux-mêmes : Dernier à boire, premier aufeu .

\section{La levée de cavaliers de l'été 1793 et le dépôt de Vienne}

Le décret de la Convention du 16 avril 1793 ordonne la levée de 30000 cavaliers armés et équipés. Ces cavaliers doivent théoriquement compléter les régiments déjà existants de dragons, hussards et chasseurs à cheval de l'armée du Rhin. Ce premier décret de la Convention est complété par un second, le 27 juin 1793, qui fixe à 1/10e du contingent de l'infanterie des départements celui des hommes désignés pour la cavalerie, autrement dit 30000 cavaliers. Le décret du 22 juillet 1793 fixe le nombre de cavaliers à trois cent quarante-cinq pour le département de l'Ain. D'après ce décret, les communes doivent fournir à chaque cavalier un sarrau et un pantalon de toile ou de coutil, un bonnet de police, un col noir, trois chemises, deux paires de souliers, deux paires de bas et un sac de toile. Les hommes composant ces levées successives sont généralement jeunes. Sur les vingt-huit recrues du contingent du district de Châtillon, l'âge moyen est de 23 ans et demi. Tous mesurent entre 5 pieds 2 pouces et 5 pieds 6 pouces. Si certains sont dirigés sur Valence, ceux du district de Châtillon-surChalaronne partent tous le 2 octobre 1793 accompagnés d'un officier pour rejoindre Vesoul, garnison d'un régiment de dragons (1).

Dès le 26 juin 1793, la Convention prévoit qu'une partie de ces hommes, une partie des cavaliers jacobins (équipés par les sociétés populaires), joints aux troupes de la Légion germanique et des hussards de la Liberté, forment le $11^{\mathrm{e}}$ régiment de hussards. Néanmoins, une partie d'entre eux est dirigée sur le dépôt «La Patriote » de Vienne, où ils restent sans emploi jusqu'à la création des hussards des Alpes, futur $13^{\mathrm{e}}$ régiment de l'arme.

(1) A.D. Ain, série 5L, État des hommes au nombre de 28 faisant le contingent des recrues de cavalerie du District de Châtillon, 2 octobre 1793. 
Durant l'automne et l'hiver 1793-1794, le cloître de Brou, à Bourg abrite le ler régiment de hussards (2). Bien que leur ferveur révolutionnaire ait été jugée douteuse le $1^{\mathrm{er}}$ nivôse an II par le représentant du peuple Gouly, les hussards du $1^{\text {er }}$ régiment se montrent d'un grand zèle quand il s'agit de seconder les sans-culottes de Bourg dans leurs expéditions rurales, tant et si bien, que le jour de leur départ, l'agent national du district de Bourg, Rollet-Marat, demande, à la société des sans-culottes de Bourg le 21 pluviôse an II, que l'on conserve dans cette ville «les braves hussards qui se sont très bien conduits et qui méritent de la part des sans-culottes beaucoup d'égard» (3). Le $1^{\text {er }}$ régiment de hussards est alors «neuf», bien qu'il soit le plus ancien régiment de l'arme. En effet, suite à la défection d'une partie de ses effectifs, il est reconstitué par l'amalgame de cent hommes des dragons de la Montagne, appelés aussi hussards des Alpes (créés le 20 mars 1792 et réorganisés plus tard par le représentant du peuple Boisset), de deux escadrons de volontaires de cavalerie légère du Calvados, de cent hommes du $26^{\mathrm{e}}$ régiment de cavalerie et d'une compagnie de hulans-flanqueurs (4). Si le régiment est complété par un ordre de Paris pour servir à l'armée des Alpes, les représentants du peuple en mission lèvent une unité par arrêté du 14 thermidor an II sur le dépôt de la cavalerie de Vienne «La Patriote ». Ce dernier est sous la direction de l'inspecteur général Montagny, et reçoit une partie des chevaux réquisitionnés et des sabres remis par les municipalités.

\section{Un corps franc sur l'arrière de l'armée des Alpes (5)}

D'après l'arrêté des représentants Albitté et Laporte, du 14 thermidor an II relatif à la formation des compagnies de cavalerie, quatre compagnies de cavaliers du dépôt de Vienne sont organisées par le général Walther. Répartis en deux escadrons de deux compagnies chacun, on compte quatre cent dix-huit hommes, sans les officiers. Un mois plus tard, «les représentants du peuple Laporte et Reverchon, voulant débarrasser le dépôt de Vienne du chaos dans lequel il se trouvait plongé par une foule d'hommes et de chevaux qui ne rendaient aucun service à la République et qui lui occasionnaient de grandes dépenses et pour que l'armée des Alpes, totalement dénuée de cavalerie puisse compter sur ses secours effectifs qu'elle pouvait attendre des hommes du dépôt, prirent un arrêté en date du 15 fructidor

(2) Quelques années plus tard, en 1799 , les 4 e et $9^{\text {e }}$ régiments de chasseurs à cheval, ainsi que le $1^{\mathrm{er}}$ régiment de hussards cisalpin, seront en caserne à Bourg.

(3)A.D. Ain, 3 L, ancien D7 des A.C. de Bourg, Registre de délibérations de la société des sans-culottes de Bourg.

(4) C'est ainsi, que le père de Philibert Leduc, se retrouve admis officier au 1er régiment de hussards et arrive à Bourg comme officier d'état-major du général Lajolais.

(5) Ne pas confondre les hussards des Alpes avec les hussards de la Légion des Alpes, issus de la légion de l'armée du Midi, qui eux forment dès le 5 mars 1793 le 13e régiment de chasseurs à cheval. 
dernier par lequel ils créèrent 4 compagnies de hussards » (6). C'est à compter de ce jour que les compagnies sont dénommées officiellement hussards. Si les trois premières sont composées de militaires, la quatrième est formée des cavaliers fournis par les sociétés populaires (7). Les escadrons sont formés entre le 15 fructidor an II et le 4 brumaire an III. Dès le 15 fructidor an II, Laporte et Reverchon nomment six des dix-sept officiers du corps. Si la majeure partie de ces derniers « furent pris... parmi les officiers réformés du $5^{\mathrm{e}}$ régiment de cavalerie attachés alors au dépôt comme instructeurs et l'autre partie parmi des anciens officiers de cavalerie capables de perfectionner l'instruction de ces quatre compagnies et de les conduire au combat » (8), les sous-officiers « furent choisis dans le dépôt parmi les soldats qui ont été reconnus pour avoir déjà servi dans la cavalerie » (9). Parmi les officiers, on compte alors seize militaires, quatre paysans, quatre artisans, quatre commerçants, un avocat et quatre citoyens. L'aire de recrutement géographique est variée; si l'on trouve plusieurs Rhodaniens, il y a aussi des Normands et des Alsaciens. D'où qu'ils viennent, ils acquièrent très rapidement l'esprit de corps. Si, le 3 vendémiaire an III, leur formation est approuvée, l'organisation du corps semble plutôt inexistante.

\section{Une organisation difficile}

Le 4 vendémiaire an III, les représentants du peuple Albitte et Saliceti, de Loano, nomment provisoirement Guérin, vicomte d'Etoquigny (10), chef d'escadron des hussards des Alpes avec mission d'organiser et d'uniformiser l'unité. Ce dernier, capitaine de dragons et plus ancien adjoint des adjudants généraux de l'état-major de l'armée des Alpes, est alors chargé par Walther d'organiser le grand dépôt de cavalerie de Vienne. Rapidement, Guérin se met à l'œuvre et rencontre beaucoup de difficultés pour former son unité. En effet, non seulement les hommes sont éparpillés dans les dépôts de Lyon, Mâcon et Bourg (11), mais les représentants du peuple à Lyon font appel à

(6) S.H.A.T., XL 260, Lettre des officiers et sous-officiers des hussards des Alpes à la commission d'organisation des mouvements de l'armée de Terre, 5 ventôse an III.

(7) Les cavaliers de ce contingent proviennent essentiellement de Lyon. Il est à noter que les sociétés populaires de Saint-Rambert et Ambérieu-en-Bugey, fournissent elles aussi deux cavaliers jacobins.

(8) S.H.A.T., XL 260, Lettre des officiers et sous-officiers des hussards des Alpes à la commission d'organisation des mouvements de l'armée de Terre, 5 ventôse an III.

(9) Ibid

(10) Né le 28 avril 1762 à Dieppe, il fait des études à l'École royale des Ponts et Chaussée de 1784 à 1788. Il est sous-lieutenant au $8=$ dragons le 15 septembre 1791 . Forte tête mais excellent officier, il est nommé lieutenant le 23 décembre 1792. Adjoint aux adjudants généraux à l'état-major de l'armée des Alpes, en septembre 1793, il est adjoint à l'adjudant général Charton le 15 avril 1794. C'est là qu'il est remarqué par les deux conventionnels.

(11) A.D. Ain, série 1L. Cinquante-trois hussards se trouvent à Bourg avec un capitaine, deux souslieutenant et trois maréchaux des logis. Ils ont cinquante-huit chevaux. Sur ces hommes stationnés à Bourg, deux sont à l'hôpital, un en prison, quinze sont alors garnisaires et vingt-huit servent à la correspondance dans le département. Le 12 brumaire an III, Boisset, autorise le lieutenant Simon de la 3e Compagnie à venir au dépôt à Bourg «se faire délivrer... un cheval sur le payement du prix du maximum ». 
eux, pour se rendre dans l'Ain, faire rentrer les subsistances en réquisition pour Lyon et les magasins de l'armée des Alpes. Les différents détachements envoyés dans l'Ain ne comptent que des hussards et des sous-officiers, pas de spécialistes (maréchaux-ferrants ou artistes vétérinaires). Ces cavaliers appartiennent à la $3^{\mathrm{e}}$ compagnie (12), rattachée au dépôt central de Vienne.

Habillés à la hongroise, les hussards des Alpes portent des couleurs distinctives : pelisse écarlate, dolman et culotte bleu ciel, boutons et galonnages jaunes. Ils sont coiffés d'un mirliton à longue flamme noire et bleue. Ils portent une sabretache qui bat la jambe gauche et un sabre de cavalerie légère modèle 1786 "révolutionnaire », que leur esprit crâne et élitiste commande de laisser traîner. En effet, le sabre utilisé par les hussards des Alpes peut être un sabre à monture à une seule branche en laiton. La poignée de section ovale est en bois basané. Celle-ci est terminée par une longue queue à boutons à facettes. Une paire de croisillons assujettit le sabre à son fourreau. Ce dernier est constitué, suivant le modèle 1786, d'un fût de bois recouvert de cuir et renforcé de deux grandes garnitures en laiton avec un ou deux anneaux de bélières. Avec l'économie de guerre, les garnitures en laiton sont rapidement remplacées par des garnitures en fer, moins souples, plus difficiles à travailler et surtout oxydables, fragilisant l'ensemble. C'est ainsi équipés que les hussards des Alpes arrivent dans l'Ain au moment où Guérin continue de mettre sur pied son unité.

\section{Les subsistances : \\ un enjeu crucial de la guerre et de la politique intérieure}

Dès l'été de l'an II, les réquisitions pour subvenir aux besoins de Lyon et de l'armée commencent à pleuvoir sur le département de l'Ain. Chaque district en est de son contingent de réquisitions. Le 18 floréal an II, le directoire du département de l'Ain répartit la réquisition de fourrages décidée le 25 germinal an II par la commission des subsistances : le district de Châtillon doit fournir 250 quintaux de foin et 400 quintaux de paille; celui de Montluel doit fournir 200 quintaux de foin et 400 quintaux de paille, celui de Pont-de-Vaux 250 quintaux de foin et 400 quintaux de paille et celui de Trévoux 300 quintaux de foin et 500 quintaux de paille. Le 11 messidor an II, l'agent en chef des subsistances militaires de l'armée des Alpes envoie une circulaire aux districts formant les bases arrière de l'armée des Alpes pour l'emmagasinement des avoines dans les magasins militaires. Il rappelle l'arrêté du Comité de salut public du 28 prairial an II qui stipule qu'outre la mise en réquisition de «toutes les avoines existantes dans l'étendue de la 
république,... tout possesseur d'avoine sera tenu d'en faire le versement » (13). Le 24 thermidor an II, le directoire du département répartit une seconde réquisition de foin, d'avoine et de paille pour l'armée des Alpes, en date du 12 thermidor an II, décidée par la commission des approvisionnements : le district de Pont-de-Vaux doit 3000 quintaux de foin, 3000 quintaux d'avoine et 10000 quintaux de paille, le district de Trévoux doit 4000 quintaux de foin, 12000 quintaux d'avoine et 10000 quintaux de paille, le district de Châtillon doit 4000 quintaux d'avoine et 8000 quintaux de paille et le district de Montluel doit 8000 quintaux d'avoine et 8000 quintaux de paille.

Si les premières réquisitions sont bien exécutées, il semble que le relâchement thermidorien entraîne dans les campagnes une fronde latente qui se traduit par des tendances à la désobéissance, un début d'accaparement et de spéculation. Pour les habitants des campagnes, sur qui pèsent le plus ces réquisitions, la confiance dans l'assignat est amoindrie par sa dévaluation constante (14). Alors que la loi du maximum est abrogée, ces réquisitions ne sont pas faites pour arranger les finances des agriculteurs étant donné qu'ils sont payés suivant « le taux des mercuriales » (15).

Dès le mois de thermidor an II, les difficultés d'approvisionnement se font vraiment sentir dans le district de Pont-de-Vaux. Les 17 et 18, la société populaire de Bâgé demande à la municipalité «une réquisition de bled et seigle suffisante pour l'approvisionnement des citoyens de ladite commune » (16). À l'annonce de cette pétition, l'agent national du district prend la mesure des événements. Désormais averti de la nécessité d'approvisionner les marchés, il sait aussi que la seule manière d'y parvenir est d'avoir recours aux réquisitions. Mais si « les réquisitions sans nombre qu'il [le district de Pont-de-Vaux] a essuyé l'année dernière a dépourvu absolument les citoyens de leur subsistance » (17), il sait qu'il faut les organiser et surtout bien les diriger, car «la cupidité de certains cultivateurs est telle qu'ils se refusent de conduire leurs denrées dans les marchés qu'ils sont habitués d'approvisionner, que même des officiers municipaux égoïstes et sans délicatesses se refusent de délivrer des acquits à caution à ceux qui sont bien intentionnés » (18). Sans doute profite-t-il de la présence, dans les environs de Pont-de-Vaux, de troupes à cheval, pour lever de nouvelles réquisitions le 22 thermidor an II. Les pénuries en grains sont telles que les troupes

(13) Ibid., série 10L, Circulaire de l'agent en chef des subsistances militaires de l'armée des Alpes au district de Saint-Rambert, 11 messidor an II.

(14) En nivôse an III, un assignat de 100 livres s'accepte pour 25, en pluviôse an III il est accepté pour 22 et en ventôse an III pour 20 livres.

(15) A.D. Ain, série 5L25, Délibérations du district de Châtillon, 19 nivôse an III.

(16) A.C. Bâgé, Rév.10, Séance publique du district de Pont-de-Vaux, 22 thermidor an II.

(17) Ibid.

(18) Ibid, 
à cheval n'ont pas de fourrages. Pour subvenir à la nourriture des chevaux, il faut, le 3 vendémiaire an III, que le district requiert Louis Fetus, grenadier, de faire verser au magasin 60 quintaux de foin. Désormais, tout trafic de grains prend une importance extrême. Le 11 vendémiaire an III, deux sacs d'avoine appartenant à un citoyen de Meximieux sont saisis à Bourg, tandis que le citoyen Greppo, ancien feudiste employé aux subsistances militaires à Trévoux, est suspecté par le district de Montluel. Le 17 vendémiaire an III, à la suite d'une réquisition de subsistances pour la Grenette de Montluel, des citoyens informent le district «qu'il se fait par le Rhône clandestinement et au mépris des lois une déportation de grains » (19) qui met « le district dans l'impossibilité de remplir diverses réquisitions dont il est frappé » (20). Pour contrecarrer ce trafic, le directoire du district de Montluel prend un arrêté qui établit un guet de quatre gardes nationaux et un caporal, à Thil, sur les bords du Rhône.

\section{Des hussards dans les districts dombistes et bressans de l'Ain}

Alors qu'à Vienne, Guérin bataille pour organiser ses escadrons, le directoire du district de Montluel rappelle dans son arrêté du 25 vendémiaire an III qui fixe la mission des hussards mais surtout le mode d'exécution des réquisitions, que toute exportation secrète de denrées est illégale et que les agents nationaux sont personnellement responsables des «événements qu'ils n'auront pas prévenus » (21). Pour cela, le district se réserve le droit de mettre en état d'arrestation les accapareurs, les trafiquants ainsi que toutes les personnes suspectées dans ce type d'affaire. Trois jours plus tard, le directoire du district de Pont-de-Vaux enregistre un arrêté de Boisset concernant les «mesures à prendre contre ceux qui refusent de satisfaire aux réquisitions soit pour l'armée des Alpes, Commune Affranchie et les semences orientales du département» (22). Cet arrêté concorde avec une lettre de l'agent national du district qui impute aux agriculteurs la pénurie de grains dans laquelle se trouve le district. En effet, les « habitants des campagnes... [dont] la cupidité et l'habitude qu'ils ont contractées... d'exporter dans les départements circonvoisins les bleds qui doivent servir à la subsistance des défenseurs de la Patrie et à l'approvisionnement de nos frères des districts qui n'en récoltent pas » (23) font que les divers arrêtés et les invitations fraternelles à coopérer restent lettre morte. Dans un arrêté du

(19) Ibid.

(20) Ibid

(21) Collection de l'auteur, Extrait des registres de délibérations du directoire du district de Montluel du 25 vendémiaire an III.

(22) AD. Ain, série 9L, Registre de délibérations du directoire du district de Pont-de-Vaux.

(23) Ibid 
20 brumaire an III, les représentants du peuple Ritter et Turreau, envoyés auprès de l'armée des Alpes et d'Italie, restreignent l'accès aux subsistances entreposées dans les magasins militaires «de la gauche du Rhône depuis Genève jusqu'à Arles » (24). Dans le district de Bourg, les réquisitions de subsistances posent aussi de gros problèmes. Dans plusieurs communes (25), des habitants sont condamnés, par jugement du tribunal du district de Bourg, à verser des grains dans les magasins. En outre, les communes de Marboz et de Foissiat sont obligées de cantonner et de nourrir des détachements de canonniers. Ainsi quatre des neuf districts du département de l'Ain (les plus éloignés de la frontière) vont connaître, plusieurs semaines durant, une occupation militaire qui ne prendra fin qu'avec la livraison des réquisitions demandées.

\section{Le district de Montluel}

Dès le début du mois de vendémiaire an III, un détachement de quatre hussards des Alpes, auquel est joint un gendarme «pour faire le service communal » (26), stationne à Miribel. Le 21 vendémiaire an III, le directoire du district députe un commissaire auprès des représentants du peuple à Lyon afin de demander de la troupe pour obtenir des denrées : «l'égoïsme et toutes les passions qu'enfante l'intérêt se développent avec un accroissement fatal et bientôt, si l'on n'emploie pas les mesures les plus coercitives, il n'y aura plus dans les communes... aucun grain disponible ni pour les armées ni pour les citoyens » (27) écrivent les administrateurs du directoire.

Si les hussards des Alpes sont mis à la disposition des représentants du peuple par un arrêté du 23 vendémiaire an III, dès le 22, le district de Montluel décide que quatre hussards sont « envoyés en détachement à Miribel et dans les communes avoisinantes pour y veiller à la sûreté publique»(28). Le lendemain, les conventionnels Charlier et Pocholle envoient «trente hussards du dépôt de Vienne... à Montluel pour... accélérer le versement des grains dont ce district est chargé envers la commune de Lyon, presser le battage et toutes les réquisitions relatives à la chose publique » (29). De leur caserne, les trente hussards des Alpes se mettent en mouvement pour Montluel. Deux jours après l'arrêté de Charlier et Pocholle, le 25, le district définit le mode des réquisitions pour Commune-

(24) Ibid., série 1L, arrêté de Ritter et Turreau, 20 brumaire an III.

(25) Ce sont les communes de Marboz, Foissiat, Saint-Martin-le-Châtel, Longchamp, Lent, Malafretaz et Bény.

(22) A.D. Ain, série 7L, Registre des délibérations du directoire du district de Montluel.

(27) Ibid., série 7L9, Registre de délibérations du district de Montluel, 11 vendémiaire an III.

(28) Ibid., série 7L, Registre des délibérations du directoire du district de Montluel.

(29) Collection de l'auteur, Extrait des registres de délibérations du directoire du district de Montluel du 25 vendémiaire ah III. 
affranchie qui doivent se faire « conformément à l'arrêté du représentant du peuple Boisset » (30).

Les trente hussards cantonnés à Montluel sont répartis par l'agent national du district avec mission d'arrêter et de saisir « tous grains en contravention aux lois » (31) ainsi que « les voitures à chevaux et tout ce qui servirait à masquer la fraude » (32). Sept hussards commandés par un brigadier se rendent à Loyes, où le maire leur donne leur instruction. Ils doivent surveiller la rivière d'Ain, du pont de Chazey à Villette, surtout la nuit. Un second détachement de quatre hussards part sur les bords du Rhône vers une maison appelée Le Soleil. Quatre autres hussards «voltigeront» (33) sur les rives du Rhône, à Thil, surtout la nuit. Quatre hussards commandés par un brigadier se rendent au Montellier pour patrouiller de Saint-Andréde-Corcy à Cordieux. Puis les huit autres hussards, sous les ordres d'un maréchal des logis, restent cantonnés à Montluel comme réserve. Suite à l'assassinat d'un paysan, le district décide, le 23 brumaire, d'utiliser un détachement de quatre hussards de la réserve de Montluel afin de remplacer un piquet de deux gendarmes à Crans.

Chaque détachement de hussards reçoit alors une barque et son conducteur. Ils peuvent aussi compter sur la collaboration des municipalités $\mathrm{du}$ district, qui « sont requises de donner main forte aux hussards » (34). Les maires et officiers municipaux des communes sont chargés « de leur faire connaître tous les passages et faux-fuyants favorables à la contrebande »(35). Pour légaliser l'action des hussards des Alpes dans leur district, les administrateurs remettent à chaque chef de détachement un extrait du procès verbal de la séance du 25 vendémiaire an III du directoire du district. Le 29 frimaire, Charlier, Pocholle et Letellier ordonnent une nouvelle réquisition de subsistances dans le district de Montluel. Des hussards partent à Beynost en «garnison ensuite des ordres du représentant du peuple pour accélérer le versement des grains requis pour l'armée des Alpes et Lyon » (36).

La solde des hussards est réglée par le receveur du district. De ce fait, les hussards deviennent partie intégrante du dispositif administratif du district. Pour les municipalités obligées de cantonner un détachement de hussards, l'injustice est flagrante et le 23 ventôse, la municipalité de Beynost demande comment et par qui les hussards en cantonnement dans leur ville doivent être payés. Le district, considérant que ces charges sont extraordi-

(30) Ibid.
(31) Ibid.
(32) Ibid.
(33) Ibid
(34) Ibid.
(35) Ibid.
(36) A.D. Ain, série 7L, Registre des délibérations du directoire du district de Montluel. 
naires, décide que seuls, ceux frappés de la réquisition seront propres à payer, et non les municipalités.

La mise en place du dispositif se fait sans que soit prise en compte la subsistance des hussards eux-mêmes. Les premiers confrontés à ce problème sont les quatre hussards cantonnés à Miribel qui ne parviennent pas à faire venir de Lyon une voiture de grains pour la nourriture de leur chevaux. Ils demandent alors au district de Montluel que le garde-magasin des subsistances militaires de Miribel soit autorisé à leur fournir du fourrage et de l'avoine pour leurs chevaux. Si les hussards de Miribel se sont trouvés un temps désemparés, le district de Montluel ne commet pas deux fois la même erreur : la nourriture est aux frais des logeurs (à l'exception du détachement de Loyes) et le pain est fourni par le boulanger Claude Brédy, de Montluel. Ce dernier reçoit en échange de son pain des bons du maréchal des logis, commandant le détachement, qu'il se fait rembourser auprès de l'administration du district. Le 22 brumaire, après un mois de présence, Brédy demande le paiement de 442 rations de pain livrées entre le 24 vendémiaire et le 14 brumaire.

Après quelques jours, il s'avère nécessaire de simplifier le service des hussards pour le rendre plus efficace. Le $1^{\text {er }}$ brumaire, le district réorganise le cantonnement des hussards du Pollet. En effet, si la maison Berthelon passe pour être officiellement trop éloignée « pour surveiller avec le même succès l'exportation clandestine de grains » (37), il est probable que le logement des quatre hussards gêne le propriétaire. Le district décide alors d'utiliser les biens échus à la Nation et loge les quatre hommes dans la maison des émigrés Gérando. De même, le district réorganise la distribution de nourriture. Le $1^{\text {er }}$ brumaire, il charge le magasin de Meximieux de nourrir les hommes cantonnés au Montellier et au Pollet, tandis que le magasin de Miribel se charge de ceux du Soleil, Brédy ne devant plus livrer du pain qu'aux hommes stationnés en ville.

Malgré les efforts du district pour subvenir aux besoins des hussards, la livraison de nourriture pour les montures semble toujours poser des problèmes. Le 21 ventôse, les hussards se plaignent à l'administration qu'ils n'obtiennent pas les fourrages nécessaires à leurs chevaux du citoyen Nant fils, «attendu, disait-il, qu'il en manquait lui-même » (38). Le directoire du district fait venir Nant pour l'interroger. Ce dernier annonce qu'il ne peut plus fournir de foin par manque de matière, et qu'il en achète à un citoyen de Rignieux-sur-Toison qui, malgré la promesse d'achat, n'honore pas son engagement et le revend plus cher ailleurs. Le district, reconnaissant «que les hussards cantonnés dans l'étendue du district sont de la plus grande 
utilité » (39), et conscient que la fourniture de fourrage pour les hussards est essentielle au bon fonctionnement des réquisitions, décide de faire délivrer à Rignieux, le fourrage nécessaire.

\section{Le district de Pont-de-Vaux}

En exécution des arrêtés des représentants du peuple à Lyon en date du 23 vendémiaire, un détachement de hussards, basé à Mâcon, est envoyé cantonner à Pont-de-Vaux.

La troupe, mise en garnison dans les communes, a le même rôle que celles basées dans les autres districts à la différence que les hussards détachés dans les villages de l'arrondissement sont mis sous l'autorité d'un commissaire nommé par le district «à l'effet de diriger les opérations qui lui sont confiées » (40).

Rapidement, le directoire du district de Pont-de-Vaux envoie un détachement de quinze hussards, dix gardes nationaux et un commissaire, à Manziat. Un hussard est chargé de faire quotidiennement l'estafette entre le commissaire et le district. Les administrateurs du district de Pont-de-Vaux préviennent la municipalité de Bâgé du cantonnement de troupes à Manziat «non seulement pour empêcher le passage des grains mais encore pour presser la livraison des grains requis » (41). Les administrateurs du district de Pont-de-Vaux pensent que la seule présence de cette troupe dans un canton de son arrondissement, plutôt réfractaire aux arrêtés sur les subsistances, devrait suffire. Le district demande donc aux municipaux de Bâgé de renseigner le commissaire sur la fraude des grains, l'achat clandestin d'œufs et de beurre, " afin que la force armée puisse en faire la recherche la plus exacte » (42). Le district prie aussi les officiers municipaux de Bâgé «de faire faire de fréquentes patrouilles par la garde nationale, surtout dans les charrières qui conduisent aux moulins de la Veyle, parce que c'est là précisément où se fait l'achat des grains par les Mâconnais » (43). À Manziat, le commissaire Patin charge les hussards de faire des patrouilles « tant de nuit que de jour » (44) afin de surveiller l'exportation frauduleuse et l'emmagasinement de grains. Malgré cette décision, le district de Pont-de-Vaux continue de subir la pénurie de grains, tant à cause de la mauvaise volonté de certaines communes que de celle de certains particuliers. Au début du mois de brumaire, le commandant de la garde nationale de Bâgé demande au

(39) Ibid.

(40) Collection de l'auteur, Lettre des administrateurs du district de Pont-de-Vaux aux officiers municipaux de Bâgé, 29 vendémiaire an III.

(41) Ibid.

(42) Ibid.

(43) Ibid

(44) Ibid 
district la possibilité d'envoyer dans sa commune un détachement de hussards pour l'aider «par des patrouilles fréquentes à empêcher le passage des grains » (45). Le même jour, la municipalité de Pont-de-Vaux adresse la même requête au district, arguant le manque de grains pour subvenir à l'approvisionnement des citoyens.

Le 7 brumaire, estimant qu'un seul détachement de hussards dans la campagne ne suffirait pas à empêcher le trafic des grains, les administrateurs du district de Pont-de-Vaux annoncent aux officiers municipaux de Bâgé que douze hussards sont dépêchés dans leur commune pour y cantonner. Ces hussards, chargés de patrouiller, font équipe avec six hommes de la garde nationale locale triés sur le volet (46) et nommés par la municipalité. La municipalité de Bâgé craint sans doute les débordements de ces cavaliers dont la réputation de bravache n'est plus à faire. Le district avertit les officiers municipaux : «Nous vous recommandons de faire apporter la plus grande discipline soit dans la troupe qui sera en cantonnement chez vous soit dans la garde nationale qui fera le service et d'empêcher qu'il ne soit fait aucun dégât chez les cultivateurs » (47). Il invite la municipalité de Bâgé «à nommer un membre pris dans votre sein pour veiller à la distribution qui devra être faite tant pour les subsistances pour les hommes que pour les fourrages pour les chevaux » (48). En effet, cette dernière ne disposant pas d'un magasin militaire, le district lui indique comment les hussards et leurs montures doivent être ravitaillés : «Il s'agira donc de faire délivrer du grain à un boulanger qui sera par vous désigner, lequel s'occupera de la fabrication du pain qui doit être composé de trois quarts de froment et l'autre quart de blé seigle. Chaque pain doit être du poids de trois livres et doit servir pour deux jours, il sera également donné à chaque hussard une demilivre de viande chaque jour... Quant aux fourrages il sera bottelé du foin pour quinzaine chaque botte devra peser dix-huit livres et il en sera délivré une botte pour chaque cheval ainsi qu'à chacun cinq livres d'avoine » (49). C'est un boucher désigné par la municipalité, ainsi que les communes du canton, qui livreront les denrées pour les hussards. Quant au boulanger, s'il utilise du grain de son stock pour faire le pain des hussards, ce dernier lui sera remboursé. En outre, son travail (ainsi que celui du boucher) est payé grâce à des bons délivrés par Patin, commissaire du district, qui doivent être présentés au maire de Bâgé.

(45) A.D. Ain, série 9L, Registre de délibérations du district de Pont-de-Vaux.

(46) Il doit s'agir de citoyens actifs ayant la confiance de la municipalité de Bâgé.

(47) Collection de l'auteur, Lettre des administrateurs du district de Pont-de-Vaux aux officiers municipaux de Bâgé, 7 brumaire an III.

(48) Ibid.

(49) Ibid. 
Le 9 brumaire, le directoire du district autorise les municipalités de Bâgé et de Pont-de-Vaux à se servir de la force armée cantonnée chez elles «pour faire remplir les réquisitions ordonnées par le directoire pour l'approvisionnement des marchés » (50). Le 11 brumaire, un détachement de vingt hussards du $2^{\mathrm{e}}$ escadron est cantonné chez l'aubergiste Basset de Pont-de-Vaux. Ces vingt cavaliers et leurs montures y demeurent jusqu'au 6 frimaire suivant. Dans tout le district, les hussards se mettent à l'œuvre. Leurs patrouilles, si elles fatiguent les fers des chevaux, usent aussi les sabres des hommes qui doivent faire réparer au citoyen Buisson la "garniture d'un sabre » (51) et raccommoder la garniture en cuir de plusieurs (52).

Les craintes des administrateurs du district de Pont-de-Vaux de voir des exactions militaires se commettre se révèlent justes, notamment entre Bâgé et Saint-Laurent-sur-Saône, là où les difficultés de la surveillance devaient être les plus intenses. Des habitants de Saint-Laurent se plaignent de l'attitude des hussards cantonnés à Bâgé. Le 18 brumaire, les officiers municipaux portent plainte auprès de leurs collègues de Bâgé contre cinq hussards, qui « arrêtoient sur les différentes routes, et même jusqu'à la faire gâcher, le beurre, œufs, volailles et qu'ils commettoient plusieurs excès, comme de prendre la dite volaille à des prix fort bas... de prendre même le beurre et jusqu'au linge qui couvrent les paniers » (53). Ces troubles suscitent rapidement des disputes à main armée avec les autochtones qui, s'ils sont un moment impressionnés, réagissent rapidement et ne veulent plus se laisser faire. Désirant mettre fin à ces troubles, les officiers municipaux de Saint-Laurent invitent les officiers municipaux de Bâgé « à réunir vos efforts au nôtre afin de contenir les 5 hussards dans notre district» (54). L'attitude des hussards est aussi dénoncée par les municipaux de Saint-Laurent au commissaire des guerres de Mâcon.

Malgré tout, le district de Pont-de-Vaux continue de faire confiance à la présence dissuasive des hussards et prend même un arrêté qui nomme deux nouveaux commissaires pour parcourir le canton de Saint-Trivier, afin de « forcer les réfractaires à s'exécuter » $(55)$ et leur adjoint à « chacun un détachement d'hussards pour protéger leur opération » (56). Peu de temps après que les commissaires se soient mis en route accompagnés par les hussards, les résultats se font sentir. Le 23 brumaire, les commissaires se plaignent auprès du directoire du district, de l'agent national et d'un officier municipal de Marsonnas qui retiennent le tableau de recensement des grains. Tandis

(50) A.D. Ain, série 9L, Registre de délibérations du district de Pont-de-Vaux.

(51) Ibid.

(52) Buisson touchera dix livres du payeur de la guerre pour ce travail.

(53) Lettre des officiers municipaux de Saint-Laurent-sur-Saône aux officiers municipaux de Bâgé, 18 brumaire an III, A.D. Ain, 108J.

(54) Ibid.

(55) Registre de délibérations du directoire du district de Pont-de-Vaux, ibid, série 9L.

(56) Ibid. 
que le directoire du district décide de les faire arrêter par les hussards et la gendarmerie. Les hussards cantonnés à Pont-de-Vaux quittent la ville. Le voiturier Galland est requis par le district pour emmener les bagages du détachement à Mâcon (57). Le lendemain, ce sont les hussards du détachement de Bâgé qui reçoivent du district l'ordre de se rendre en cantonnement à Saint-Laurent-sur-Saône, où la municipalité désirait avoir de la troupe pour surveiller les accaparements.

\section{Les districts de Trévoux et de Châtillon}

Le 1er nivôse, les compagnies de hussards sont organisées et passées en revue au dépôt «La Patriote » à Vienne, par Guérin et le conseil d'administration, et quelques jours plus tard, un détachement est envoyé de Lyon dans le district le Trévoux. Rapidement, la nécessité d'être sur le terrain amène l'inspecteur principal des subsistances à envoyer les vingt-cinq hussards en détachement à Châtillon afin d'assurer et accélérer le recensement des grains. Les hussards des Alpes sous les ordres d'un brigadier et d'un maréchal des logis arrivent, le 2 nivôse an III, à Châtillon où ils sont cantonnés. Là, le district leur délivre des bons et des mandats pour régler leurs dépenses courantes, comprenant la solde, l'entretien des montures et la ration de légumes. Le brigadier sert d'ordonnance entre Châtillon et Trévoux. Mais, le 15 nivôse, le commandant du détachement prévient le district que ses hommes partiront pour Trévoux. Alors qu'il annonce au district le départ de la totalité du détachement, il se rend compte qu'il est nécessaire de maintenir sur place une partie de la garnison; seuls douze hussards se rendent à Trévoux. Ils ont pour mission d'accompagner les divers commissaires nommés par le district pour réquisitionner les subsistances. Si le détachement du district de Trévoux est sous les ordres du maréchal des logis Allinot, qui supervise toutes les opérations et les affaires ayant trait à son détachement (58), il est aussi sous l'autorité du district de Châtillon, ce qui ne va pas sans complications. En effet, en temps ordinaire la solde est réglée par le conseil d'administration du régiment, mais durant leur temps de cantonnement elle est réglée par les districts. La suprématie du détachement de Châtillon sur celui de Trévoux prévaut aussi sur la solde. En effet, le détachement est toujours attaché à celui de Châtillon et c'est à ce dernier district de régler la solde des hussards même s'ils sont à Trévoux. Ainsi, le 11 nivôse, le maréchal des logis qui commande le détachement des douze cavaliers de Trévoux écrit au district de Châtillon pour lui signifier le refus du conseil d'administration de payer la solde de ces hommes même

(57) Il reçoit 25 livres pour le travail.

(58) Il contrôle et certifie véritable le mémoire de pansement du vétérinaire Bruyères avant que celui-ci soit remis au directoire du district de Trévoux le 23 nivôse an III. 
s'ils ne sont qu'à 5 lieues de Lyon. Quatre jours plus tard, le commandant du détachement fait savoir au district de Châtillon que le quartier maître du régiment et le commissaire des guerres acceptent de régler la solde de ses hommes à condition qu'il obtienne un certificat qui l'autorise à toucher la solde des douze hommes de Trévoux.

Le détachement de Trévoux, pour faciliter ses manœuvres, est à son tour scindé en deux. Une partie est à Thoissey et l'autre à Trévoux. Les hussards du district de Trévoux patrouillent très fréquemment en utilisant les grands axes de communication, ce qui use rapidement les fers de leur chevaux. Le 13 nivôse, les maréchaux-ferrants Louis Morgon, de Thoissey, et Antoine Parcet, de Trévoux, annoncent au directoire du district qu'ils ont « consumé les fers dont ils étaient approvisionnés... pour le ferrage qui a été nécessité... pour les chevaux des hussards appelés pour les compléments de grains requis » (59). Le directoire du district de Trévoux conscient de la présence nécessaire des hussards des Alpes (60), cherche à améliorer les conditions de leur mission, en mettant à la disposition de la municipalité de Thoissey deux quintaux de fer (provenant sans doute des débris des clochers) pour permettre «de ferrer les chevaux de cavalerie» (61). Ces fers seront remis, au fur et à mesure des besoins, aux maréchaux-ferrants. Une mesure similaire est adoptée pour les maréchaux-ferrants de la municipalité de Trévoux. Malgré le soin apporté aux montures et à leurs fers, certains animaux se blessent.

Les hussards en garnison dans le district de Trévoux sont logés et nourris par les habitants et les artisans de la ville. Quotidiennement, les hussards de Trévoux reçoivent de la viande du boucher Lacroix tandis que les chevaux, eux, reçoivent du foin. Les dépenses de nourriture prises en charge par l'administration se révèlent lourdes. Les autorisations de paiement, délivrées sur bons ou justificatifs par le directoire du district de Trévoux, sont réglées par le receveur du district sur les fonds destinés aux frais de guerre. Mais, le 11 nivôse, à l'image de la solde, le règlement des subsistances des hussards du district de Trévoux, et de leurs montures, s'avère aussi du ressort du district de Châtillon qui doit alors rembourser l'administration du district de Trévoux. Le maréchal des logis du détachement de Trévoux fait parvenir au district de Châtillon le livret des dépenses de son détachement afin que ce dernier paie la solde et rembourse aussi les frais de subsistance et de fourrage. Dès lors, la nourriture qui est fournie par les habitants du district de Trévoux est payée par le receveur du district suivant le maximum, ce qui ne convient pas au boucher Lacroix qui demande, le 23 nivôse an III,

(59) A.D. Ain, série 11L, Registre de délibérations du directoire du district de Trévoux, 13 nivôse an III.

(60) Le Directoire, considérant qu'il importe de veiller à tout ce qui peut concourir à l'entretien des troupes requises pour quelque service que ce soit, ibid.

(61) Ibid. 
une augmentation du prix de la viande qu'il livre chaque jour aux hussards. Dans sa séance quotidienne, le directoire du district de Trévoux fixe alors le prix de la livre de viande fournie par Lacroix, à 26 sous. Par contre, le citoyen Bion reçoit, (suivant le maximum), 13 livres en paiement des 260 livres de foin fournies aux hussards et, le 23, l'expert vétérinaire Bruyères, qui réclame 60 livres pour «le pansement et le traitement fait à un cheval de hussard » (62), ne reçoit que 40 livres.

Dans le district de Châtillon, les patrouilles de hussards sont très fréquentes et efficaces. Leur présence incite plusieurs municipalités à faire appel à eux. Le 10 nivôse, alors que le maréchal des logis commandant le détachement est en pleine tractation avec le district de Châtillon pour le règlement des frais du détachement du district de Trévoux, la municipalité de Saint-Cyr-sur-Menthon dénonce quatre citoyens qui refusent de livrer leurs grains. Alors que la municipalité demande au district d'envoyer quatre ou cinq hussards pour forcer l'exécution des réquisitions, les municipalités de Saint-Julien et de Laiz dénoncent à leur tour le retard de six et quinze citoyens respectivement, et demandent aussi l'aide des hussards des Alpes. Le 12, le district décide d'envoyer quatre hussards, nommés par le maréchal des logis, à Saint-Cyr. Sept jours plus tard, le district envoie quatre hussards à Laiz et quatre autres à Saint-Julien. Les hussards sont sous l'autorité des municipalités afin de faire exécuter le recensement des grains mais aussi les réquisitions. Ils sont logés et nourris aux frais des municipalités qui sont autorisées à obtenir le remboursement de leurs frais par les citoyens en retard.

Les détachements cantonnés dans le district de Châtillon ne partent que le 26 nivôse an III. Leur entretien coûte 355 livres 12 sols et 51 fers à cheval sont changés, ce qui atteste des fréquents déplacements de cette force armée.

\section{Utilité des hussards dans les réquisitions?}

La présence des hussards des Alpes dans l'Ain est un succès à court terme puisque les subsistances requises pour Lyon sont livrées. Mais, d'une manière générale, leur venue rencontre un échec à moyen terme : «l'audace des malfaiteurs s'accroît de jour en jour par l'impunité et l'absence d'une force suffisante pour les réprimer et les poursuivre » (63). En effet, avec les nouvelles réquisitions, pour approvisionner les marchés des villes de l'Ain, de Bourg notamment, les habitants des campagnes continuent d'accaparer les grains malgré une récolte très fructueuse pour l'an III. L'avertissement qu'ont voulu donner les représentants du peuple et les administrateurs des

(62) A.D. Ain, 11L, Registre de délibérations du directoire du district de Trévoux, 23 nivôse an III.

(63) Ibid., 7L, Registre des délibérations du directoire du district de Montluel. 
districts aux récalcitrants n'a eu qu'un effet temporaire. Dès le mois de thermidor an III, la municipalité de Bourg se plaint des « laboureurs (qui) refusent à livrer, à un prix honnête, les denrées les plus nécessaires à la subsistance » (64). Malgré les réquisitions et les arrêtés du directoire du département, les agriculteurs résistent : le 18 messidor an III, le district de Châtillon doit faire incarcérer un laboureur qui a refusé de conduire une ânée de froment à la grenette. Le 15 fructidor, seize municipalités du district de Bourg sont dénoncées comme n'ayant pas accompli les réquisitions et le 30 brumaire an IV, le directoire du département envoie une force armée dans quatre communes qui refusent de fournir du blé à la grenette de Bourg. Faut-il en déduire qu'il y a alors deux types de réquisitions, une jugée de première importance qui concerne les armées pour lesquelles on emploie des moyens extraordinaires et une deuxième jugée de moindre importance qui concerne les communes, les marchés et les grenettes locales? Étant donné les efforts déployés par les représentants du peuple pour fournir les réquisitions militaires et l'efficacité avec laquelle elles sont appliquées, on peut sans nul doute penser que oui.

En outre, le passage des hussards des Alpes dans l'Ain ne reste pas sans conséquences. Alexandre Basset, aubergiste à Pont-de-Vaux écrit au maire de la ville, en floréal an VIII, pour obtenir le paiement de la dette occasionnée par le casernement des hussards chez lui cinq ans auparavant; s'il a été payé pour le logement des hommes, il n'a pas reçu les 2 sols par jour et par cheval auxquels il avait droit.

\section{Les hussards des Alpes à l'armée des Alpes}

L'arrivée de ces troupes à Vienne pose de nouveaux problèmes à Guérin, puisqu'il doit les équiper et uniformiser leur tenue. Guérin garde les distinctions uniformologiques des dragons des Montagnes, ossature des hussards des Alpes. Après beaucoup d'efforts, le régiment prend tournure : «Les officiers et sous-officiers se livrèrent sans réserve à l'instruction de leurs compagnies respectives, qui furent dès lors mises sous les ordres du général en chef de l'armée des Alpes, pour être employées comme il le jugeait le plus utile pour le bien de la chose publique. Depuis 7 mois, notre instruction a été poussée avec la dernière activité. Nous ne craignons pas de dire que ces deux escadrons peuvent s'en tirer avec honneur devant l'ennemi » (65). Le 31 janvier 1795 (nivôse an III), le corps est officiellement

(64) Plainte des officiers municipaux de Bourg du 7 thermidor an III, citée par E. DUBOIS dans Histoire de la Révolution dans l'Ain, tome 5, p. 46.

(65) S.H.A.T., XL 260, Lettre des officiers et sous-officiers des hussards des Alpes à la commission d'organisation des mouvements de l'armée de Terre, 5 ventôse an III. 
formé sur les arrières de l'armée des Alpes. Malgré le départ d'Albitte, de Saliceti, de Reverchon et de Laporte, et l'emploi d'une partie de l'unité dans l'Ain, Cassanyès continue de nommer les officiers du corps. Le 4 brumaire, ce sont neuf officiers, dont un aide de camp du général Lajollais, qui sont nommés aux hussards des Alpes, malgré un avis favorable qui avait été donné pour des officiers venant de l'unité déjà existante, suivant les directives de Dubois-Crancé acceptées par la Convention le 3 vendémiaire an III. Ces nominations d'officiers extérieurs au corps, qui découlent de l'amalgame, déplaisent aux cadres des quatre compagnies de hussards qui s'en plaignent à Paris le 5 ventôse : «Pendant cette intervalle deux lieutenances se sont présentées dans ces quatre compagnies, nous espérions qu'elles seraient remplies par les officiers et les sous-officiers du corps, qui, seuls avaient contribué à l'instruction de ces compagnies et que l'élection s'en ferait conformément à la Loi ; mais nos espérances ont été trompées. Les fruits de nos travaux ont été recueillis par des étrangers au corps » (66). Malgré cette protestation, les nominations de Cassanyès prennent effet.

Devant la nécessité de former une unité de cavalerie légère, Cassanyès, de Grenoble, signe le 12 pluviôse an III un arrêté qui enrégimente, de manière provisoire les quatre compagnies de hussards du dépôt de Vienne, formées d'une compagnie de dragons de la Montagne (dits hussards des Alpes), plus l'excédent de la compagnie des guides à cheval de l'Armée des Alpes, mélangé à une compagnie de cavaliers dont le noyau est pris dans toutes les autres en dépôt à Vienne. Le régiment est formé à partir de ces quatre compagnies. Lidée de former un nouveau régiment « avec d'autres compagnies détachées que nous ne connaissons pas encore » (67) ne plaît pas à l'encadrement des compagnies de hussards qui, en voyant les deux lieutenances confiées à des étrangers au corps, disent on ne «nous a laissé que douleurs pour récompense de nos travaux » (68). Même si certains hommes sont enrégimentés depuis les mois d'avril à août 1793, les effectifs ne cessent de se renouveler ou de se renforcer de floréal an III à nivôse an IV. Les hussards sont originaires de toute la France et sont, en principe presque tous des anciens soldats (69) ; ainsi on trouve des hommes venant de Lyon, de Montélimar, de Montpellier mais aussi de Nevers, de Strasbourg, de Schelestadt, de Laon, de Paris, de Vannes, d'Aix ou de Rennes.

Le nouveau régiment des hussards des Alpes est composé de deux escadrons, comprenant chacun 2 compagnies. Chacune est commandée par un capitaine qui a sous ses ordres un lieutenant, deux sous-lieutenants, un

(66) Ibid.

(67) Ibid,

(fig) Ibid.

(69) Parmi l'encadrement, on trouve quinze militaires sur trente-trois officiers. Les autres sont des civils, dont six agriculteurs, cinq artisans, deux commis, trois étudiants et un avocat. 
maréchal des logis chef, deux maréchaux des logis, un brigadier fourrier, quatre brigadiers et cinquante-huit hussards, dont quatre à pied (70).

Comme tout régiment, les hussards des Alpes ont un étendard. Plusieurs hypothèses sont envisageables quant au modèle. D'abord, on peut supposer l'utilisation d'un étendard ayant en son centre un dragon tenant dans ses pattes avant un ruban sur lequel est écrit vigilentia hussards, avec au-dessous et en dessous les légendes de 1791 : discipline I obéissance I à la loi. Dans chaque angle figure un faisceau de licteurs au bonnet rouge et au milieu de chaque côté, une foudre avec éclairs dans une couronne de lauriers, le tout relié par des feuillages et des perles. Le fond est blanc et la bordure tricolore. Mais les hussards des Alpes ont peut être aussi utilisé le modèle du colonel Levasseur Dumont : fond blanc avec bordure or ou tricolore, avec au centre le numéro du régiment (ici 13) entouré d'une couronne de feuillages, avec en haut, un ruban portant discipline / obéissance I à la loi, en bas, hussards de la République. Dans les angles du haut figurent un macaron rouge et un bleu portant des feuillages au centre desquels se trouve un bonnet phrygien traversé par un ruban portant, l'un liberté et l'autre ou la mort. Dans les angles du bas, figurent les mêmes macarons aux mêmes motifs mais portant citoyens et incorruptibles.

Ce qui peut être sûr, c'est que, dès l'an II, il est prévu que les régiments de hussards portent des guidons. Régiment de volontaires composé de compagnies issues de plusieurs formations, celui-ci utilise sans doute les guidons des différentes compagnies formant les hussards des Alpes.

Ayant réussi à former une unité en état de combattre, Guérin est nommé chef de brigade provisoire des hussards des Alpes le 12 pluviôse an III par le représentant du peuple Cassanyès. Le 15 fructidor an III, les hussards des Alpes deviennent le $13^{\mathrm{e}}$ régiment de hussards.

\section{Le 13e hussard à l'armée des Alpes}

Guérin reste à la tête de ses hussards jusqu'au 10 brumaire an IV, date à laquelle, après avoir été suspecté de royalisme, il est remplacé par l'ancien chef de brigade du $21^{\mathrm{e}}$ régiment de chasseurs à cheval, Landrieux. Le $13^{\mathrm{e}}$ hussard ne compte alors pas moins de trois dépôts; Toulon, pour la troupe, commandé par un capitaine et un sous-lieutenant, Castres, pour la remonte, et Nice où se trouvent le magasin du régiment, mais aussi l'hôpital, commandés par cinq sous-lieutenants et un capitaine. C'est dans ce dernier dépôt que se trouve l'artiste-vétérinaire. Répartis dans ces trois dépôts, ne se trouvent pas moins de cent quatre-vingt-seize hommes et quatre-vingt- 
seize chevaux. Avant même de combattre, le $13^{\mathrm{e}}$ hussards éprouve des pertes non négligeables : disparition d'un capitaine à Aix le 24 nivôse an IV, disparition du payeur des escadrons de guerre à Nîmes en pluviôse et disparition d'un maréchal des logis et d'un brigadier au Pont-du-Var le 28 ventôse. Durant cette période, un quartier maître trésorier, un adjudant et un sous-lieutenant disparaissent ou désertent du dépôt de Nice le 2 germinal an IV.

Désormais formé en unité militaire, le $13^{\mathrm{e}}$ régiment de hussards est à l'armée d'Italie. Bien que les hommes ne touchent pas l'argent de la masse d'entretien, depuis vendémiaire an IV, les engagements ne cessent pas : six des vingt-deux hussards décédés ou disparus, se sont engagés après l'enrégimentement des hussards des Alpes. Dix d'entre eux sont dans le régiment depuis 1793. Le 13e hussard subit rapidement des pertes dues aux combats mais aussi à des accidents ou des soulèvements ruraux : le 16 germinal an IV, un brigadier meurt après être tombé dans la mer avec son cheval à Saint-Mauricio, le 20 germinal an IV, un hussard est tué par des paysans à Orméa. Le 22 germinal, un escadron rejoint le général Joubert à Ceva, où il sert à l'avant-garde du corps du Pontévalloi. Dès leur arrivée à Ceva, un hussard décède dans une rixe. Il faut attendre le 24, pour que le reste du régiment entre dans la division Kilmaine, en compagnie du $7^{\mathrm{e}}$ bis de hussards, le $24 \mathrm{e}$ régiment de chasseurs à cheval, les $8^{\mathrm{e}}$ et $15^{\mathrm{e}}$ régiments de dragons. Ils forment la $2^{\mathrm{e}}$ division de cavalerie. Ce n'est qu'à cette date que Landrieux rejoint le régiment à Aix. De là, le régiment se porte sur Alba. À la suite de la prise de la redoute de Mondovi, un hussard décède à l'hôpital le 4 floréal an IV. Le 8 mai, le $13^{\mathrm{e}}$ hussard passe le Pô sur des barques jumelées. Il est envoyé à Pizzighettone. Le 10, il est aux combats de Mollev et de Cadayno où il se comporte avec vaillance. Alors que le 12, il passe par Malazzano pour déboucher, le 13, à Lodi où se trouve le quartier général, le hussard Alexis Dufour est tué par des paysans à Asti. Même s'il est au bivouac, le régiment continue d'éprouver des pertes : trois hussards et un brigadier meurent à Fombio et un brigadier disparaît à Codogno le 19. Le lendemain, un hussard se noie à Picighetone et un autre est tué. Le 24 encore, un hussard est tué à Mattéo.

Malgré tout, le $13^{\mathrm{e}}$ hussard donne une bonne image de lui, puisque Bonaparte compte quelques hussards de ce régiment attaché à son étatmajor (71). C'est durant la bataille de Lodi, le 21 floréal, que le $13^{\mathrm{e}}$ hussard se couvre de gloire. Bien que le moral de la troupe soit élevé (72), comme dans tous les régiments de hussards, et que la solde soit perçue régulièrement, Landrieux, qui avait déjà été suspendu de son poste au 21e chasseurs pour sa mauvaise gestion du régiment, s'attire les inimitiés de ses officiers.

(71) Bonaparte et son état-major en 1796, tableau d'Édouard DETAILLE, Musée de l'Armée, Paris.

(72) Même si le 24 floréal, à Lodi, un capitaine donne sa démission. 
Et, bien que les généraux soient satisfaits du régiment et de sa bonne tenue (seul un brigadier déserte à l'ennemi à Alba le 16 floréal an IV), Landrieux écrit, vexé de voir ses hommes se prononcer contre lui, au Général Lacuée, pour demander le licenciement du régiment.

Sur ordre du général Berthier, le général de division Édouard Kilmaine, accompagné du commissaire des guerres Roux, se rend, le 29 floréal, au bivouac du $13^{\mathrm{e}}$ hussard situé dans la prairie de Lodi. Arrivés au bivouac, les deux hommes font appeler Landrieux. Kilmaine s'adresse à lui : «Je lui ai notifié lesdits ordres portant que le corps qu'il commande sera à l'instant incorporé moitié dans le $1^{\text {er }}$ et moitié dans le $7^{\mathrm{e}}$ régiment de hussards » (73) et que les vingt cinq hommes, «les mieux montés » (74), passent dans les Guides de l'Armée des Alpes. Cette incorporation dans les Guides prouve la qualité du régiment (75). Puis, Kilmaine, accompagné de Roux et Landrieux, passe le régiment en revue. Sur un effectif de trois cent trenteneuf hussards et sous-officiers, seuls cent soixante-huit sont présents (76). De même, sur les vingt-huit officiers du régiment, seule une petite partie est présente. Si beaucoup sont dans les dépôts, un sous-lieutenant est en permission et un lieutenant en mission à Vienne. Le régiment est néanmoins dissous : soixante-quatre des hommes présents, «montés, armées et équipés » (77) ainsi que quatre-vingt-neuf hommes du dépôt de Nice et seize de Castres, passent au 1er hussard. Cinquante-neuf des hussards présents montés et équipés, vingt démontés ainsi que les quatre-vingt-sept de Toulon et les quatre derniers de Castres passent au $7^{\mathrm{e}}$ hussard (78). Landrieux, avec sept officiers, passe au $7^{\mathrm{e}}$ hussard tandis que huit autres passent au 1er (79) et deux obtiennent de servir comme commissaire des guerres. Le médecin $\mathrm{du}$ régiment et son aide passent au 15 e régiment de dragons. Le $13^{\mathrm{e}}$ hussard n'existe plus.

(73) S.H.A.T., XC 260, procès-verbal d'incorporation du $13^{\mathrm{e}}$ régiment de hussards dans les $1^{\mathrm{er}}$ et 7e hussards.

(74) Ibid

(75) Le hussard Michel, de Digne, engagé à 15 ans au $13^{\mathrm{e}}$ hussard entre dans les guides en prairial an IV et finit sa carrière comme maréchal de camp en 1835.

(76) 123 sont montés, 20 ne le sont pas et 25 ont incorporés les Guides sans passer la revue.

(77) S.H.A.T., XC 260, procès-verbal d'incorporation du 13e régiment de hussards dans les ler et 7e hussards.

(78) Le procès-verbal mentionne bien le $7^{\mathfrak{e}}$ hussards alors qu'à l'époque c'est le $7^{\mathrm{e}}$ hussards bis qui est à l'armée d'Italie.

(79) Le lieutenant Bérougne de Juniac, qui passe capitaine au 13e hussard, se retrouve au $1^{\mathrm{er}}$ suite à la dissolution du régiment. Il devint une gloire de ce régiment qu'il commanda par ailleurs. 


\section{Conclusion}

L'utilisation d'un corps de hussards, afin de faire réquisitionner les subsistances pour Lyon et l'armée des Alpes est une nouveauté dans les événements révolutionnaires de l'Ain. En effet, l'appel à ces hussards marque une volonté nouvelle dans la conduite de l'effort de guerre qui n'est plus l'apanage d'une armée de soldats-citoyens mais celle d'hommes d'armes obéissant à des supérieurs hiérarchiques. Si politiquement, s'en est bientôt fini de l'agitation populaire, il en est de même militairement, les volontaires sont devenus de véritables guerriers, qui déplacent l'idéologie républicaine de 1793 vers l'obéissance à un chef qui leur apporte victoire, gloire, honneur et nourriture. 